\title{
Effects of Edaravone on Muscle Atrophy and Locomotor Function in Patients with Ischemic Stroke
}

\section{A Randomized Controlled Pilot Study}

\author{
Hiroaki Naritomi, ${ }^{1}$ Hiroshi Moriwaki, ${ }^{2}$ Norifumi Metoki, ${ }^{3}$ Hiroyuki Nishimura, ${ }^{4}$ \\ Yasuto Higashi, ${ }^{5}$ Yasumasa Yamamoto, ${ }^{6}$ Hiroyuki Yuasa, ${ }^{7}$ Hiroshi Oe, ${ }_{1}^{1}$ Kortaro Tanaka, ${ }^{8}$ \\ Kozue Saito, ${ }^{2}$ Yasuo Terayama, ${ }^{9}$ Tadafumi Oda, ${ }^{10}$ Norio Tanahashi ${ }^{11}$ and Hisao Kondo, ${ }^{12}$ \\ on behalf of the MARVELOUS (Muscular Atrophy Restraint with Vigilant Edaravone \\ Long-term Use after Stroke) Study Group \\ 1 Senri Chuo Hospital, Toyonaka, Japan \\ 2 National Cerebral and Cardiovascular Center, Suita, Japan \\ 3 Hirosaki Stroke Center, Hirosaki, Japan \\ 4 Nishinomiya Kyoritsu Neurosurgical Hospital, Nishinomiya, Japan \\ 5 Himeji Central Hospital, Himeji, Japan \\ 6 Kyoto Second Red Cross Hospital, Kyoto, Japan \\ 7 Tosei General Hospital, Seto, Japan \\ 8 Toyama University, Toyama, Japan \\ 9 Iwate Medical University, Morioka, Japan \\ 10 Saito Yukoukai Hospital, Ibaraki, Japan \\ 11 Saitama Medical University International Medical Center, Hidaka, Japan \\ 12 Kyushu University, Fukuoka, Japan
}

Background and Objective: Stroke patients with severe leg paralysis are often bedridden in the acute and subacute phase, which increases the risk of disuse muscle atrophy in the chronic phase. The evidence to date indicates that oxidative stress plays an important role in the mechanism of disuse muscle atrophy. Therefore, the aim of this study was to determine if long-term radical scavenger treatment with edaravone following an acute stroke prevents the progression of disuse muscle atrophy and improves leg locomotor function in the chronic phase.

Methods: This randomized controlled pilot study was conducted at 19 acute stroke and rehabilitation centers across Japan. Forty-seven ischemic stroke patients with at least leg motor weakness admitted within 24 hours of onset were randomly assigned to receive continuous intravenous infusions of edaravone $30 \mathrm{mg}$ twice daily for 3 days (short-term group) or 10-14 days (long-term group). The primary endpoints of the study included the degree of 
leg disuse muscle atrophy, as measured by the percentage change from baseline in femoral muscle circumference $15 \mathrm{~cm}$ above the knee, and the improvement in leg locomotor function, as assessed by the maximum walking speed over $10 \mathrm{~m}, 3$ months after the onset of stroke.

Results: Three-month follow-up was completed by a total of 41 patients ( 21 in the short-term group and 20 in the long-term group). On admission, there was no significant difference in the severity of stroke or the grade of leg paresis between the two treatment groups. The grade of disuse muscle atrophy and incidence of gait impairment 3 weeks after stroke onset were also similar between the short- and long-term groups. However, disuse muscle atrophy of the paretic and non-paretic legs was significantly less severe in the long-term versus the short-term treatment group $(3.6 \pm 5.9 \%$ and $1.5 \pm 6.0 \%$ vs $8.3 \pm 5.2 \%$ and $5.7 \pm 6.4 \% ; \mathrm{p}<0.01$ and $\mathrm{p}<0.05) 3$ months after stroke onset. Additionally, the maximum walking speed over a distance of $10 \mathrm{~m}$ was significantly greater in the long-term group ( $98 \pm 67$ vs $54 \pm 55 \mathrm{~cm} / \mathrm{sec} ; \mathrm{p}<0.05)$.

Conclusion: Edaravone treatment for up to 14 days suppresses the progression of disuse muscle atrophy and improves leg locomotor function to a greater extent than shorter-term treatment in acute stroke patients. This suggests that the management of stroke may be improved with long-term edaravone therapy by providing myoprotective effects that ameliorate functional outcome in the chronic phase.

\section{Introduction}

Stroke is a global health problem and is a major cause of death and disability in the adult population. ${ }^{[1]}$ One of the most common symptoms of stroke is motor weakness, which can lead to considerable disruption of activities in daily life such as feeding, bathing, dressing, and grooming. During a cerebral infarction, motor tracts in the frontal cortex, the corona radiata, the internal capsule, or the pons are often impaired, resulting in the development of hemiparesis of the lower limbs and subsequent leg locomotor dysfunction during the chronic phase of stroke. ${ }^{[2]}$

However, leg locomotor dysfunction is not solely attributable to ischemic cerebral damage of motor neuron tracts, but may also be in part due to disuse muscle atrophy of both paralyzed and non-paralyzed legs. Indeed, a small study of healthy older adult subjects demonstrated that 10 consecutive days of bed rest caused substantial losses in skeletal muscle mass and function, par- ticularly in the lower extremities. ${ }^{[3]}$ Acute stroke patients with severe hemiparesis or paralysis can remain bedridden for at least 1-2 weeks, and are often unable to walk despite weeks of rehabilitation. This extended period in a bedridden state during the acute and subacute phases of stroke is the likely cause of lower limb disuse muscle atrophy in association with leg motor weakness, thus accelerating the development of leg locomotor dysfunction in the chronic phase. ${ }^{[4,5]}$

The involvement of oxidative stress in disuse muscle atrophy was first highlighted in the 1990s. ${ }^{[6-8]}$ Although the exact mechanisms of disuse muscle atrophy in humans remain unclear, the evidence to date has established the important role that oxidative stress plays in the development of disuse muscle atrophy. ${ }^{[9,10]}$ The free radical scavenger edaravone is a neuroprotective drug that has been indicated for the treatment of acute stroke since 2001 in Japan, and has been shown to dose dependently improve functional outcomes in patients with acute ischemic stroke. ${ }^{[1-13]}$ Edaravone 
can donate an electron to a lipid peroxyl radical, which results in conversion to a lipid peroxyl anion. ${ }^{[14]}$ Edaravone scavenges free radicals produced by activation of the arachidonic acid cascade during cerebral ischemia-reperfusion, and inhibits the peroxidation of lipids by free radicals. ${ }^{[14]}$ Edaravone has been shown to protect the vascular endothelium and reduce damage to neuronal and glial cells by scavenging for free radicals. ${ }^{[14-17]}$

In Japan, the recommended duration of edaravone administration is 14 days, but this can be shortened in patients with mild symptoms. ${ }^{[18]}$ Early studies indicated that neuronal cells cannot survive for more than several hours after ischemia and that cell-killing events continue for a few days. These studies suggest that neuroprotective drugs may exert protective effects if started within several hours after ischemia and continued for a few days. ${ }^{[19,20]}$ Based on these findings, a 3-day administration of edaravone is considered sufficient to exert adequate cerebroprotection. However, Unno et al. ${ }^{[12]}$ reported that the duration of edaravone therapy affected the functional outcome in acute ischemic stroke patients. Moreover, animal studies indicate that the process of radical-related disuse muscle atrophy begins several days after leg immobilization. ${ }^{[6-10]}$ This suggests that radical scavenger therapy should be administered for a longer period when used to suppress the development of radical-related disuse muscle atrophy.

The aim of the present pilot study entitled MARVELOUS (Muscular Atrophy Restraint with Vigilant Edaravone Long-term Use after Stroke) was to determine if long-term treatment with the free radical scavenger edaravone can (i) suppress disuse muscle atrophy following an acute stroke; and (ii) alleviate the degree of gait impairment during the chronic phase.

\section{Patients and Methods}

\section{Patients}

Nonconsecutive acute stroke patients admitted to an acute stroke center within 24 hours of onset were eligible for the study. Inclusion criteria were age 20-79 years, the presence of a leg motor weakness rating of 2-4 on the National Institute of Health Stroke Scale (NIHSS) motor subscore on admission, and no apparent functional disability prior to stroke as estimated by a modified Rankin Scale (mRS) score of 0 or 1. Exclusion criteria were a history of hypersensitivity to edaravone, associated neuromuscular diseases, obstructive femoral artery diseases, congestive heart failure, significant renal/hepatic diseases, hemorrhagic stroke, use of thrombolytic therapy, a Japan Coma Scale score of 20 or more, history of neurologic dysfunction with an mRS score of $\geq 2$, artificial knee or hip joints, serum creatinine $>1.5 \mathrm{mg} / \mathrm{dL}$, malignant disorders, pregnancy, or enrollment in another clinical study.

The study was approved by the local ethics committee of each participating institute and was conducted in accordance with the World Medical Association Declaration of Helsinki and the Ethical Guidelines for Epidemiological Research of the Japanese Ministry of Education, Culture, Sports, Science and Technology, and the Japanese Ministry of Health, Labour and Welfare. All patients provided written informed consent prior to entry into the study and were centrally registered via the Internet.

\section{Study Design}

The study was an open-label, randomized, controlled pilot study that was conducted in 19 institutes comprising 13 acute stroke centers and six rehabilitation centers in Japan. A central assignment/registration method via the Internet was used for case registration and allocation; preregistered individual identification and password was used for verification. Input included the time of hospital admission, name of the hospital, age, sex, severity (an NIHSS motor subscore of the hemiparetic lower limb), and name of the person who input the data.

Randomization was performed with control factors of the name of the hospital, age, sex, and an NIHSS motor subscore of the hemiparetic lower limb, and the cases were divided into two groups with a ratio of $1: 1$. Patients were randomized to receive edaravone ( $30 \mathrm{mg}$ twice daily), adminis- 
tered intravenously for 3 days (short-term group) or 10-14 days (long-term group).

All patients were admitted to an acute stroke center and underwent bedside rehabilitation therapy, followed by out-of-bed rehabilitation therapy. The start time of rehabilitation therapy was classified into four categories: (i) on the day of admission; (ii) within 3 days of admission; (iii) within 7 days of admission; and (iv) 8 or more days after admission. Following the completion of acute-phase rehabilitation therapy, patients were transferred to a post-stroke rehabilitation center.

It should be noted that physicians from the acute stroke center were responsible for follow-up evaluations of leg muscle volume and locomotor function at 3 weeks and/or 3 months post-stroke in patients who were transferred to a rehabilitation center that was not affiliated with the MARVELOUS Study Group. In contrast, patients transferred to a MARVELOUS-affiliated rehabilitation center were evaluated by a physician from the rehabilitation center.

\section{Procedures}

Edaravone (Radicut $^{\circledR}$, Mitsubishi Tanabe Pharma Corporation, Osaka, Japan) administration was started upon admission. Patients received edaravone $30 \mathrm{mg}$ twice daily, which was dissolved in $100 \mathrm{~mL}$ of saline and administered by continuous intravenous infusion for 30 minutes. Edaravone was administered for 3 consecutive days in the short-term group and 10-14 consecutive days in the long-term group.

\section{Assessment of Study Endpoints}

The primary endpoints of the study included changes from baseline in the degree of leg disuse muscle atrophy and the severity of leg locomotor dysfunction 3 months after the onset of stroke. Leg disuse muscle atrophy was defined as a reduction (expressed as a percentage) from baseline (within 4 days of admission) in femoral muscle volume at 3 weeks and 3 months after the onset of stroke, which was assessed by measuring the femoral muscle circumference of both legs at 5 , 10 , and $15 \mathrm{~cm}$ above the knee when patients were in a supine position with both legs outstretched. The severity of locomotor dysfunction was evaluated based on the Brunnstrom Recovery Stage $(\mathrm{BRS})^{[21]}$ and maximum walking speed (MWS) over a distance of $10 \mathrm{~m}$.

\section{Statistical Analysis}

According to unpublished data from our preliminary study of ischemic stroke patients undergoing edaravone treatment, 'good' locomotor function (mRS 0-2) 3 months after stroke onset was seen in $17 \%$ and $60 \%$ of patients receiving 3 and 14 days of edaravone therapy, respectively. Based on these findings, sample size calculations using a power of $80 \%$ indicated that a minimum of 38 patients was required to detect a difference in the primary endpoint, assuming a two-sided significance level of 0.05 .

Data were expressed as percentages for categorical variables or means for continuous variables. A Pearson's chi-squared $\left(\chi^{2}\right)$ or Fisher's exact test was used for the statistical comparison of categorical variables. An unpaired Student's t-test was also used to compare differences in the degree of disuse muscle atrophy and MWS between the two groups. Furthermore, simple regression analysis was undertaken to assess the correlation between the degree of paralyzed leg muscle atrophy and MWS. A p-value of $<0.05$ was considered to be statistically significant. Statistical analyses were carried out using Microsoft ${ }^{\circledR}$ Office Excel 2003.

\section{Results}

A total of 47 eligible patients were registered into the study during the 1 -year period from 1 January 2007 to 31 December 2007. Three-month follow-up was successfully completed by 41 of 47 patients; data from these 41 patients were included in the analysis. The six patients who did not complete the 3-month follow-up were excluded from the analysis for the following reasons: the development of cerebral hemorrhage during the follow-up period $(\mathrm{n}=1)$; discontinuation of edaravone due to the occurrence of liver disorders during treatment $(\mathrm{n}=1)$; inability to 
continue the drug infusion for more than 1 day because of heart failure $(n=1)$; no rehabilitation was performed during the follow-up period due to associated pneumonia and other complications $(n=1)$; and discontinuation of follow-up for personal reasons $(n=2)$. Consequently, the analysis was performed in 21 patients in the short-term ( 3 days of edaravone therapy) group and 20 patients in the long-term (10-14 days of treatment) group.

Table I shows a detailed summary of patient characteristics of the two study groups. There was no significant difference in age, sex, risk factors, stroke subtypes, severity of stroke as defined by the NIHSS score, or the grade of leg paresis on the affected side as defined by the NIHSS motor subscore. All patients in the short-term group received 3 days of edaravone treatment; the mean \pm standard deviation duration of edaravone treatment in the long-term group was $13.6 \pm 1.2$ days.

Table II compares the state of rehabilitation therapy in the acute phase, the incidence of gait disability 3 weeks after stroke onset, and the severity of leg disuse muscle atrophy 3 weeks after stroke onset between the short- and long-term edaravone treatment groups. The time of initiation of bedside and out-of-bed rehabilitation therapy and the total days of rehabilitation were similar between the two treatment groups. Gait disability 3 weeks after stroke onset was defined as confinement to a wheelchair or a bedridden state, and was reported in $47.6 \%$ and $45.0 \%$ of patients in the short- and long-term groups, respectively. The severity of disuse muscular atrophy in the paretic and non-paretic legs (measured $15 \mathrm{~cm}$ above the knee) was numerically greater in the short-term group $(5.0 \pm 3.4 \%$ and $3.7 \pm 4.4 \%$, respectively), compared with the long-term group $(4.4 \pm 4.1 \%$ and $2.0 \pm 3.8 \%$, respectively) 3 weeks after stroke onset, but this difference was not statistically significant.

The effect of edaravone treatment on the grade of leg disuse muscle atrophy and the level of leg locomotor function during the chronic phase ( 3 months after the onset of stroke) is summarized in table III. The grade of disuse muscle atrophy (i.e. percentage reduction from baseline in femoral muscle volume) in the paretic and nonparetic legs at $15 \mathrm{~cm}$ above the knee was signif-

Table I. Patient characteristics ${ }^{a}$

\begin{tabular}{|c|c|c|}
\hline Characteristic & $\begin{array}{l}\text { Short-term edaravone } \\
(n=21)\end{array}$ & $\begin{array}{l}\text { Long-term edaravone } \\
(\mathrm{n}=20)\end{array}$ \\
\hline$\overline{\text { Age }(y)[\text { mean } \pm S D]}$ & $70.0 \pm 7.3$ & $69.2 \pm 7.5$ \\
\hline $\operatorname{Sex}(n, M / F)$ & $13 / 8$ & $14 / 6$ \\
\hline \multicolumn{3}{|l|}{ Risk factors [n (\%)] } \\
\hline hypertension & $17(80.9)$ & $16(80.0)$ \\
\hline diabetes mellitus & $8(38.1)$ & $7(35.0)$ \\
\hline dyslipidemia & 7 (33.3) & $8(40.0)$ \\
\hline atrial fibrillation & $3(14.3)$ & $5(25.0)$ \\
\hline \multicolumn{3}{|l|}{ Stroke subtype [n (\%)] } \\
\hline cardioembolic & $3(14.3)$ & $4(20.0)$ \\
\hline atherothrombotic & $13(61.9)$ & $12(60.0)$ \\
\hline lacunar & $5(23.8)$ & $4(20.0)$ \\
\hline Duration of study treatment (days) [mean \pm SD] & $3.0 \pm 0.0$ & $13.6 \pm 1.2$ \\
\hline NIHSS score on admission [median (range)] & $7(3-24)$ & $9(2-19)$ \\
\hline NIHSS motor subscores [median (range)] & $3.0(2-4)$ & $2.5(2-4)$ \\
\hline Severe leg weakness ${ }^{\mathrm{b}}[\mathrm{n}(\%)]$ & $11(52.4)$ & $10(50.0)$ \\
\hline \multicolumn{3}{|l|}{ a Data for 41 patients who completed the study. } \\
\hline \multicolumn{3}{|c|}{ b Severe leg weakness was defined as an NIHSS motor subscore of 3-4. } \\
\hline \multicolumn{3}{|c|}{$\mathbf{F}=$ female $\mathbf{M}=$ male $; \mathbf{N I H S S}=$ National Institute of Health Stroke Scale. } \\
\hline
\end{tabular}


Table II. Acute-phase rehabilitation, gait disability, and the severity of disuse muscle atrophy 3 weeks after the onset of stroke ${ }^{a}$

\begin{tabular}{|c|c|c|}
\hline & $\begin{array}{l}\text { Short-term edaravone } \\
(\mathrm{n}=21)\end{array}$ & $\begin{array}{l}\text { Long-term edaravone } \\
(\mathrm{n}=20)\end{array}$ \\
\hline \multicolumn{3}{|l|}{ Acute-phase rehabilitation } \\
\hline bedside within 3 days of admission [n (\%)] & $14(66.7)$ & $14(70.0)$ \\
\hline out-of-bed within 7 days of admission [n (\%)] & 7 (33.3) & $11(55.0)$ \\
\hline total duration (days) & $16.0 \pm 5.0$ & $16.4 \pm 7.3$ \\
\hline Gait disability 3 weeks after stroke onset [n (\%)] & $10(47.6)$ & $9(45.0)$ \\
\hline \multicolumn{3}{|l|}{ Disuse muscle atrophy $(\%)^{\mathrm{c}}$} \\
\hline paretic leg & $5.0 \pm 3.4$ & $4.4 \pm 4.1$ \\
\hline non-paretic leg & $3.7 \pm 4.4$ & $2.0 \pm 3.8$ \\
\hline \multicolumn{3}{|c|}{ a Values are expressed as mean \pm standard deviation unless stated otherwise. } \\
\hline \multicolumn{3}{|c|}{$\begin{array}{l}\text { b Gait disability was defined as patients in a wheelchair or bedridden state, with a National Institute of Health Stroke Scale (NIHSS) motor } \\
\text { subscore of 3-4. }\end{array}$} \\
\hline \multicolumn{3}{|c|}{$\begin{array}{l}\text { c Disuse muscle atrophy was expressed as a mean percentage reduction from baseline in femoral muscle volume (i.e. muscle circumference } \\
\text { measured } 15 \mathrm{~cm} \text { above the knee). }\end{array}$} \\
\hline
\end{tabular}

icantly less severe in the long-term group, compared with the short-term group $(3.6 \pm 5.9 \%$ and $1.5 \pm 6.0 \%$ vs $8.3 \pm 5.2 \%$ and $5.7 \pm 6.4 \% ; \mathrm{p}<0.01$ and $\mathrm{p}<0.05$ ). A similar trend in disuse muscle atrophy was also seen when assessed at 5 and $10 \mathrm{~cm}$ above the knee (data not shown).

Long-term treatment with edaravone was associated with a significant improvement in leg locomotor function, as measured by MWS $(97.9 \pm 67.3$ vs $53.6 \pm 54.8 \mathrm{~cm} / \mathrm{sec} ; \mathrm{p}<0.05$ vs the short-term group). A larger proportion of patients in the long-term group also reported good leg locomotor function than the short-term group (70.0\% vs $57.1 \%$ ), as defined by a BRS of $5-6$, but this difference was not statistically significant. Finally, there was a significant negative correlation between the severity of disuse muscle atro- phy in the paretic leg and MWS $(r=-0.87$; $\mathrm{p}<0.001)$ [figure 1].

\section{Discussion}

The free radical scavenger edaravone is currently the only cerebroprotective agent to be approved for clinical use in the management of acute stroke, and has been shown to improve functional outcome in Japanese patients with acute ischemic stroke. ${ }^{[1-13]}$ During the last decade of the 20th century, at least 30 substances with cerebroprotective action were subjected to phase III clinical trials of acute stroke patients; these studies produced negative results on improved clinical efficacy with the exception of edaravone. ${ }^{[22-24]}$ However, the clinical data on edaravone should

Table III. Severity of disuse muscle atrophy and level of leg locomotor function 3 months after the onset of stroke ${ }^{a}$

\begin{tabular}{llc}
\hline & $\begin{array}{l}\text { Short-term edaravone } \\
(\mathrm{n}=21)\end{array}$ & $\begin{array}{l}\text { Long-term edaravone } \\
(\mathrm{n}=20)\end{array}$ \\
\hline Disuse muscle atrophy $(\%)^{\mathrm{b}}$ & & $3.6 \pm 5.9^{* *}$ \\
Paretic leg & $8.3 \pm 5.2$ & $1.5 \pm 6.0^{*}$ \\
Non-paretic leg & $5.7 \pm 6.4$ & \\
Level of leg locomotor function & & $14(70.0)$ \\
Patients with BRS 5-6 [n (\%)] & $12(57.1)$ & $97.9 \pm 67.3^{*}$ \\
MWS (cm/sec) & $53.6 \pm 54.8$ & \\
\hline
\end{tabular}

a Values are expressed as mean \pm standard deviation unless stated otherwise.

b Disuse muscle atrophy was expressed as a mean percentage reduction from baseline in femoral muscle volume (i.e. muscle circumference measured $15 \mathrm{~cm}$ above the knee).

BRS $=$ Brunnstrom Recovery Stage; MWS $=$ maximum walking speed; ${ }^{*} p<0.05$ vs short-term group, ${ }^{* *} p<0.01$ vs short-term group. 


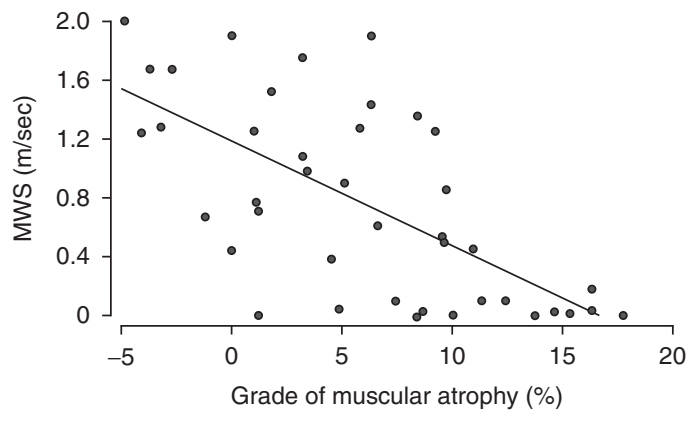

Fig. 1. Relationship between the severity of muscular atrophy in the paretic leg and maximum walking speed (MWS). MWS decreases in correlation with increases in the grade of the paralyzed leg atrophy $(r=-0.87 ; p<0.001)$. Thus, the atrophy provides significant negative effects on the walking ability.

be interpreted with caution due to a number of major differences in the design of the trial conducted on edaravone, compared with those of other cerebroprotective agents. First, edaravone could be administered within 72 hours of stroke onset, but this was restricted to within 6-12 hours of onset in studies involving other cerebroprotective agents. Secondly, the infarct size was generally smaller in the edaravone study, which resulted in minor neurologic symptoms such as motor weakness; various higher cortical dysfunctions occurred in other studies that included patients with larger infarcts. Finally, edaravone could be administered for a duration of up to 14 days, compared with 1-3 days in other studies such as the SAINT (Stroke Acute Ischemic NXY-059 [Cerovive] Treatment) II trial. ${ }^{[25]}$

The results of the phase III clinical study on edaravone suggest that the beneficial effects of the drug may also be partly attributable to myoprotective effects. ${ }^{[11]}$ Based on this hypothesis, we conducted the present pilot study to determine the myoprotective effects of edaravone in acute stroke patients. In the present study, patients were randomly allocated to edaravone therapy for a 3- or 14-day duration; this latter duration of administration was thought to be sufficient to provide both cerebroprotective and myoprotective effects.

Disuse muscle atrophy is a phenomenon that is seen in clinical practice when patients are in a long-term bedridden state or during prolonged rest with restricted activity and/or mobility. The atrophy of skeletal muscle arises from the reduction or atrophy of myofibers. ${ }^{[9,10]}$ Although the exact mechanism of onset of disuse muscle atrophy remains unclear, we reported experimental results that indicated a close relationship between oxidative stress and disuse muscle atrophy. ${ }^{[6-8]}$ More specifically, atrophic muscles of rats showed elevated levels of thiobarbituric acid reactive substances and oxidized glutathione, which are both markers of oxidative stress. Furthermore, an increase in the formation of superoxide radical anions $\left(\mathrm{O}_{2}^{-}\right)$was indicated by elevated $\mathrm{Cu} / \mathrm{Zn}$ containing superoxide dismutase activity in the cytoplasm of atrophic muscle cells, and 12 days of disuse atrophy showed a 2.2-fold rise in xanthine oxidase levels relative to controls. Indeed, histochemical studies using transmission electron microscopy revealed elevated levels of reactive oxygen species such as $\mathrm{H}_{2} \mathrm{O}_{2}$ in atrophic muscles. ${ }^{[6-8]}$ Metals such as iron have also been shown to increase oxidative stress in atrophic muscle, and the administration of the iron-chelating agent deferoxamine suppressed the increase in thiobarbituric acid reactive substances and oxidized glutathione in the atrophic muscles of rats. ${ }^{[26]}$ In recent years, the role of iron in oxidative stress has attracted close attention in the field of physiology. ${ }^{[9,10]}$

Another factor that may be involved in the development of muscular atrophy is the elevation of intracellular calcium ion $\left(\mathrm{Ca}^{2+}\right)$ levels during muscle inactivity. ${ }^{[6-8]} \mathrm{Ca}^{2+}$ levels in the cytoplasm of atrophic muscle cells were approximately 4-fold higher than levels in normal muscle cells. This accumulation of intracellular $\mathrm{Ca}^{2+}$ during muscular disuse may occur due to ionic disturbances of the cell membrane, which retards cellular removal of $\mathrm{Ca}^{2+}$. This resulting elevation of cytosolic $\mathrm{Ca}^{2+}$ may lead to the activation of calcium-dependent proteases, thus leading to the breakdown of muscle tissue.

In addition to inducing muscle atrophy during disuse, oxidative stress has been shown to be enhanced during recovery from atrophy in rats. ${ }^{[27]}$ This suggests that the production of free radicals will continue to occur in patients who are 
recovering from disuse muscle atrophy. In the present study, the continuous administration of the free radical scavenger edaravone for an extended period of 14 days was beneficial in patients undergoing disuse muscle therapy, many of whom started out-of-bed rehabilitation following several days of bed rest. Therefore, a 2-week treatment period of edaravone appears to be effective during both the period of muscle disuse and the period of recovery of muscle activity.

The severity of muscle atrophy did not differ markedly between the two treatment groups 3 weeks after stroke onset in the present study, but showed a significant improvement in favor of the long-term treatment group after 3 months. This may be explained by the delayed involvement of apoptosis during the course of disuse muscle atrophy, which eventually leads to muscular cell shrinkage and/or death. ${ }^{[9,10]}$ As a result, the clinical manifestation of muscle atrophy may not become apparent until changes in the muscular intracellular environment have taken place, which may occur over a period of several weeks.

The limitations of the present study include its small sample size and lack of a placebo or control group (i.e. standard therapy minus edaravone). Another limitation was the inability to independently assess the cerebroprotective and myoprotective effects of edaravone. An experimental study utilizing a rat middle cerebral artery occlusion model reported cellular oxidative damage in the rat brain is evident for at least 7 days. ${ }^{[28]}$ Furthermore, the time to blood reflow in humans with cerebral infarction varied according to the occluded blood vessel and stroke subtype. ${ }^{[29]}$ These studies suggest the duration of the disturbance caused by oxidative stress varies, and there is a possibility that the observed benefits on disuse muscle atrophy in the long-term group may be partly attributable to a more potent cerebroprotective effect of edaravone. If this is indeed the case, then the widely accepted view that the process of ischemic neuronal cell death is almost completed within 2-3 days of stroke onset should be reconsidered, and further clinical research investigating the administration of cerebroprotective agents for longer periods should be conducted.

\section{Conclusion}

The results of our study indicated that treatment with the free radical scavenger edaravone for 14 days effectively improved both the severity of disuse muscle atrophy and leg locomotor dysfunction in the chronic phase. Based on these findings, the use of antioxidant therapy for as long as possible appears to be warranted to provide the maximal level of both cerebroprotection and myoprotection. Larger, randomized controlled clinical trials are required to confirm the beneficial effects of edaravone in the management of stroke.

\section{Acknowledgments}

This study was funded by a Grant-in-Aid (H-18-ChojyuIppan-040) from the Ministry of Health, Labour and Welfare, Japan. The authors have no conflicts of interest that are directly relevant to the content of this study. Medical writing assistance was provided by Maxwell Chang of inScience Communications, a Wolters Kluwer business. This assistance was funded by Mitsubishi Tanabe Pharma Corporation.

The affiliations of the investigators for the MARVELOUS Study Group are as follows: Senri Chuo Hospital (H. Naritomi, H. Oe); National Cerebral and Cardiovascular Center (H. Moriwaki, K. Saito); Hirosaki Stroke Center (N. Metoki); Nishinomiya Kyoritsu Neurosurgical Hospital (H. Nishimura); Himeji Central Hospital (Y. Higashi); Kyoto Second Red Cross Hospital (Y. Yamamoto); Tosei General Hospital (H. Yuasa); Toyama University (K. Tanaka); Iwate Medical University (Y. Tarayama); Saitama Medical University International Medical Center (N. Tanahashi); Tokyo Saiseikai Central Hospital (M. Takagi); Research Institute for Brain and Blood Vessels Akita (K. Nagata); Saito Yukoukai Hospital (T. Oda); Akita Prefectural Rehabilitation and Psychiatric Center (E. Yokoyama); Toyama Koshi Rehabilitation Hospital (Y. Inoue); Hyogo College of Medicine (K. Domen); Saitama Medical School (M. Majima); Iwate Rehabilitation Center (A. Takahashi); Kyushu University (H. Kondo).

\section{References}

1. Donnan GA, Fisher M, Macleod M, et al. Stroke. Lancet 2008 May 10; 371 (9624): 1612-23

2. Schneider R, Gautier JC. Leg weakness due to stroke: site of lesions, weakness patterns and causes. Brain 1994 Apr; 117 (Pt 2): 347-54

3. Kortebein P, Ferrando A, Lombeida J, et al. Effect of 10 days of bed rest on skeletal muscle in healthy older adults. JAMA 2007 Apr 25; 297 (16): 1772-4

4. Kondo K, Ota T. Changes with time in cross-sectional areas of leg muscles in early stroke rehabilitation patients: disuse muscle atrophy and its recovery. Jpn J Rehabil Med 1997; 34: $124-33$ 
5. Odajima N, Ishiai S, Okiyama R, et al. CT findings of leg muscles in the hemiplegics due to cerebrovascular accidents: correlation to disuse atrophy. Rinsho Shinkeigaku 1987 Sep; 27 (9): 1154-62

6. Kondo H. Oxidative stress in skeletal muscle atrophy induced by immobilization. In: Reznick AZ, editor. Oxidative stress in skeletal muscle. Basel: Birkhauser, 1998: 197-13

7. Kondo H. Oxidative stress in muscular atrophy. In: Sen CK, Hanninen O, editors. Handbook of oxidants and antioxidants in exercise. Amsterdam: Elsevier, 2000: 631-53

8. Kondo H, Itokawa Y. Oxidative stress in muscular atrophy. In: Sen CK, Packer L, Hanninen O, editors. Exercise and oxygen toxicity. Amsterdam: Elsevier, 1994: 319-42

9. Powers SK, Kavazis AN, DeRuisseau KC. Mechanisms of disuse muscle atrophy: role of oxidative stress. Am J Physiol Regul Integr Comp Physiol 2005 Feb; 288 (2): R337-44

10. Powers SK, Kavazis AN, McClung JM. Oxidative stress and disuse muscle atrophy. J Appl Physiol 2007 Jun; 102 (6): 2389-97

11. Edaravone Acute Infarction Study Group. Effect of a novel free radical scavenger, edaravone (MCI-186), on acute brain infarction: randomized, placebo-controlled, double-blind study at multicenters. Cerebrovasc Dis 2003; 15 (3): 222-9

12. Unno Y, Katayama M, Shimizu H. Does functional outcome in acute ischaemic stroke patients correlate with the amount of free-radical scavenger treatment? A retrospective study of edaravone therapy. Clin Drug Investig 2010; 30 (3): 143-55

13. Lapchak PA. A critical assessment of edaravone acute ischemic stroke efficacy trials: is edaravone an effective neuroprotective therapy? Expert Opin Pharmacother 2010; 11 (10): 1753-63

14. Yamamoto Y, Kuwahara T, Watanabe K. Antioxidant activity of 3-methyl-1-phenyl-2-pyrazolin-5-one. Redox Rep 1996; 2: 333-8

15. Lee BJ, Egi Y, van Leyen K, et al. Edaravone, a free radical scavenger, protects components of the neurovascular unit against oxidative stress in vitro. Brain Res 2010 Jan 11; 1307: $22-7$

16. Ueno Y, Zhang N, Miyamoto N, et al. Edaravone attenuates white matter lesions through endothelial protection in a rat chronic hypoperfusion model. Neuroscience 2009 Aug 18; 162 (2): 317-27

17. Yamamoto T, Yuki S, Watanabe T, et al. Delayed neuronal death prevented by inhibition of increased hydroxyl radical formation in a transient cerebral ischemia. Brain Res 1997 Jul 11; 762 (1-2): 240-2

18. Mitsubishi Tanabe Pharma Corporation. Radicut (edaravone) [Japanese prescribing information]. Osaka: Mitsubishi Tanabe Pharma Corporation, 2009

19. Astrup J, Siesjo BK, Symon L. Thresholds in cerebral ischemia: the ischemic penumbra. Stroke 1981 Nov-Dec; 12 (6): $723-5$

20. Heiss WD. Experimental evidence of ischemic thresholds and functional recovery. Stroke 1992 Nov; 23 (11): 1668-72

21. Brunnstrom S. Motor testing procedures in hemiplegia: based on sequential recovery stages. Phys Ther 1966 Apr; 46 (4): $357-75$

22. Dorman PJ, Counsell CE, Sanderrock PAG. Recently developed neuroprotective therapies for acute stroke: a qualitative systematic review of clinical trials. CNS Drugs 1996; 5 (6): 457-74

23. Ginsberg MD. Neuroprotection for ischemic stroke: past, present and future. Neuropharmacology 2008 Sep; 55 (3): 363-89

24. O'Collins VE, Macleod MR, Donnan GA, et al. 1,026 experimental treatments in acute stroke. Ann Neurol 2006 Mar; 59 (3): 467-77

25. Shuaib A, Lees KR, Lyden P, et al. NXY-059 for the treatment of acute ischemic stroke. N Engl J Med 2007 Aug 9; 357 (6): $562-71$

26. Kondo H, Miura M, Kodama J, et al. Role of iron in oxidative stress in skeletal muscle atrophied by immobilization. Pflugers Arch 1992 Jun; 421 (2-3): 295-7

27. Kondo H, Kodama J, Kishibe T, et al. Oxidative stress during recovery from muscle atrophy. FEBS Lett $1993 \mathrm{Jul}$ 12; 326 (1-3): 189-91

28. Yamamoto Y, Yanagisawa M, Tak NW, et al. Repeated edaravone treatment reduces oxidative cell damage in rat brain induced by middle cerebral artery occlusion. Redox Rep 2009; 14 (6): 251-8

29. Houkin K, Ueno K, Tada M, et al. Arterial recanalization in an acute stage of cerebral infarction. Neurol Med Chir (Tokyo) 1987 Apr; 27 (4): 295-301

Correspondence: Dr Hiroaki Naritomi, Department of Neurology, Senri Chuo Hospital, 1-4-3, Shinsenrihigashimachi, Toyonaka, Osaka, 560-0082, Japan.

E-mail: naritomi@kyowakai.com 